\title{
Subject Index Vol. 37, 1991
}

Ammocoete 260 Amphibians 368 Amphibious habits 65 Anurans 317 Area, retinal 79 Arousal 260 Autoradiography 104 Aves 65, 79 Axial musculature 298

Binocular vision 65,79 Biomechanics 272 Brain size 85 - structures 125

Callosal neuron 1

Carnosine 168

Central nervous system 168

19th-century Vienna 252

Cerebral cortex 355

Cervical spinal cord 368

Cetacea 355

Chemoreception 215

Chinese pangolin, Manis pentadactyla 104

Comparative allometfy 85

- study 144

Cortex 144

Cranial nerves 10

Cytochrome oxidase 355

Dendritic morphology 1 Diencephalon 333 Dopamine 179 Double labeling I

EEG 144

Efferent octavolateralis nuclei 38

Eighth nerve 286

Escape 298

— response 286

Evolution 179

Eye movement 111

- structure 65

Feedforward interneurons 286 Fish behavior 272 Frog 92

Galliform birds 125 Ganglion cells 79,189 Geckos 144 Golden hamster 1 Goldfish 298 
Hippocampus 144 Holosteans 10,38 Homoplasy 333 Hummingbird 85

Immunohistochemistry 168, 179 Interneuron 298 Intracellular filling 1

Lamprey 260 Lateral-line afferents 38

- nerves 10

Ludvvig Mauthner 252

Mauthnercell 260,286,298,317

- fibers 252

- neuron 272 Medullary circuits 286 Membrane properties 286 Middle lateral-line nerve 38 Mixed synapses 286

Morphometry 85 Motion after-response 161 Motoneuron 298

Motor axon number 204

- $\quad$ - size 204

- columns, lateral and medial 368 MuUerceU 260

Neuroanatomy 252 Neuroethology 272 Neuromasts 10, 38 Nocturnal activity 65 Northern Fulmar 215 Nuclei, rabbit, vestibular 111 Nucleus isthmi 92

Octavolateralis system 38 Oculomotor system 111 Olfaction 215 Ophthalmology 252 Optic nerve 189

- tectum 92

Parallel homoplasy 179 Passeriform birds 125 Phylogeny 10 Physostigmine 144 Pit lines 10 Pretectum 333 Prey catching 92 Prey-predator interactions 272 Primary motoneurons 368 
Primate 189

Principal-component analysis 85 Procellariiform(es) 65,79,215 Pujjfinus pufflnus 79

Reticulospinal system 260,272 Retina 79,189 Retinal projection 104

Retinofugal projections 333 Retinopetal projections 333

Schematic eye 65 Secondary motoneurons 368 Sexual polymorphisms 204 Sharp waves 144 Spinal cord 298

- networks 298 Spiracular organ(s) 10,38 Startle 298

- behavior 260

- $\quad$ response 317 Stereotaxic brain atlas 215 Substance P 179 Swimming 298

Tectal neurons 161 Tectum 333 Telencephalon 333 Teleost(s) 204,333 Textured background 161 Thalamocortical projection 355 Threat avoidance 92 Toads 161 Topography 189 Triturus 168 Turtles 144

Urodeles 317

Vestibuloocularreflex 111 Vision 65, 79 Visual behaivor 92

- cortex 1

- $\quad$ field(s) 79

- $\quad$ streak 189 Vocalizations 204 Volumetric comparison 125 